\title{
Oligoclonal free light chains in cerebrospinal fluid as markers of intrathecal inflammation. Comparison with oligoclonal IgG
}

\author{
David Zeman $^{\mathrm{a}, \mathrm{b}, \mathrm{c}}$, Pavel Hradilek ${ }^{\mathrm{b}}$, Pavlina Kusnierova ${ }^{\mathrm{a}, \mathrm{c}}$, Radim Piza $^{\mathrm{b}}$, Katarina Reguliova ${ }^{\mathrm{b}}$, Ivana Woznicova ${ }^{\mathrm{b}}$, \\ Olga Zapletalovab
}

\begin{abstract}
Aims. To compare the sensitivity and specificity of CSF-restricted oligoclonal lgG and free light chains as markers of multiple sclerosis and other inflammatory neurological diseases.

Methods. 196 paired CSF and serum samples were examined for oligoclonal lgG and oligoclonal free light chains. The sensitivity and specificity of the tests were calculated and optimal cut-offs for the number of CSF-restricted oligoclonal bands were then determined by analysis of receiver operating characteristic curves.

Results. Optimal cut-off values were $\geq 5 \mathrm{lgG}$ bands for multiple sclerosis, $\geq 4 \mathrm{lgG}$ bands for inflammatory neurological disease, $\geq 6$ free $k$, and $\geq 2$ free $\lambda$ bands for both purposes. Using these cut-off values, sensitivities and specificities for multiple sclerosis were $83.8 \%$ and $91.3 \%$ for $\operatorname{lgG}, 83.8 \%$ and $81.0 \%$ for free $\mathrm{K}$, and $67.6 \%$ and $75.4 \%$ for free $\lambda$. For inflammatory neurological disease, sensitivities and specificities were $60.8 \%$ and $95.7 \%$ for $\operatorname{lgG}, 69.6 \%$ and $92.6 \%$ for free $\mathrm{K}$, and $64.8 \%$ and $86.2 \%$ for free $\lambda$.

Conclusions. Although exact cut-off values may vary according to method, reporting borderline results as positive, may compromise the specificity of the test and should be avoided.. The detection of intrathecal free light chain synthesis may be of value especially when the oligoclonal lgG test is negative or borderline, even though its specificity is slightly lower.
\end{abstract}

Key words: cerebrospinal fluid, oligoclonal free light chains, oligoclonal lgG

Received: May 29, 2014; Accepted: November 6, 2014; Available online: December 5, 2014

http://dx.doi.org/10.5507/bp.2014.058

aDepartment of Biomedical Sciences, Faculty of Medicine, University of Ostrava, Czech Republic

${ }^{b}$ Clinic of Neurology, University Hospital Ostrava

'Department of Clinical Biochemistry, Institute of Laboratory Medicine, University Hospital Ostrava

Corresponding author: David Zeman, e-mail: david.zeman@osu.cz

\section{INTRODUCTION}

Intrathecal synthesis of $\operatorname{IgG}$ as well as free light chains (fLC) are detectable in the majority of patients with multiple sclerosis (MS) and less frequently in other, mostly inflammatory, nervous system diseases ${ }^{1,2}$. Little information is available however that compares these tests and the existing results are somewhat conflicting.

In their seminal paper on oligoclonal free light chains (o-fLC), Sindic and Laterre reported a method of affinity-mediated immunoblotting (AIB) after isoelectric focusing (IEF) and they found that CSF-restricted free $\kappa$ light chains can occur even in the absence of oligoclonal IgG (o-IgG) in MS (ref. $\left.{ }^{3}\right)$. This was later confirmed by the same group, showing o-free $\kappa$ bands in 18 out of 33 o-IgG negative patients who presented isolated symptoms suggestive of MS (ref. ${ }^{4}$ ). Lamers et al. reported o-free $\kappa$ in most samples with CSF-restricted o-IgG and only rarely in those without ${ }^{5}$. Similar results were reported by Krakauer et al. ${ }^{6}$ using classical passive blotting but prolonged incubation (overnight) with anti-fLC antisera. These authors found o-free $\lambda$ to be both less sensitive and less specific for MS than o-free $\mathrm{k}$ which was at variance with the earlier reports of Bracco et al. ${ }^{7}$ and Gallo et al. ${ }^{8,9}$ of o-free $\lambda$ being more frequent than o-free $\kappa$ both in MS (ref. ${ }^{7,8}$ ) and human imunodeficiency virus type 1-infected patients 9 . Lolli et al. studied o-fLC by means of the classical blotting technique; they found o-fLC less frequently than o-IgG $\left(\right.$ ref. $\left.^{10}\right)$. Vakaet and Thompson used polyacrylamide gel electrophoresis followed by immunoblotting and also found o-fLC less frequently than o-IgG in MS (ref. ${ }^{11}$ ) and other inflammatory nervous system diseases ${ }^{12}$.

Positivity criteria for the o-fLC test are not well established. Usually, $\geq 2$ cerebrospinal fluid (CSF)restricted oligoclonal bands (OCB) are required for o-IgG (ref. ${ }^{1,2,13}$ ). However, some authors use different criteria for o-IgG positivity. Mayringer et al. ${ }^{14}$ reported 3 bands, which were found to be an optimum cut-off in the context of MS diagnosis in a recent large study ${ }^{15}$. Wurster reported 2 or 3 bands to be a "borderline" finding and used 4 bands as a cut-off value for definitely positive samples ${ }^{16}$. On the other hand, only 1 CSF-restricted band was considered sufficient for o-IgG positivity in another recent study ${ }^{17}$.

We therefore decided to analyze o-IgG as well as ofLC in various disease groups and to compare the sensitivity and specificity of individual tests for MS and inflammatory nervous system diseases (IND) diagnosis in general. 


\section{MATERIALS AND METHODS}

The study was approved by Ostrava University Hospital Ethics Committee (reference number 615/2011). All patients underwent lumbar puncture (LP) for diagnostic purposes; o-IgG test was always requested by the clinician. All gave written informed consent for the use of the surplus of biological material for research purposes. We examined a total of 196 paired CSF and serum samples for o-IgG and o-fLC. Subsequently, neurologists were asked to provide a diagnosis and samples were then divided into the following groups:

Group I - Multiple sclerosis (MS) at the time of lumbar puncture $(n=28)$ : mainly patients with relapsing-remitting MS $(n=21)$; only 5 patients had primary progressive MS and 2 patients secondary progressive MS;

Group II - Clinically isolated syndrome (CIS) $(n=42)$ : we simplified the classification of Miller et al. ${ }^{18}$ and grouped patients with at least one asymptomatic MRI lesion versus those without;

Group III - CNS infectious diseases $(n=13)$ : neuroborreliosis $(n=3)$, varicella zoster virus $(\mathrm{VZV})$ infection $(n=2)$, aseptic meningitis/meningoencephalitis of unknown aetiology $(n=4)$, and 1 case each of sepsis complicated by meningitis, enteroviral meningitis, herpes simplex encephalitis, and tick-borne encephalitis;

Group IV - Other inflammatory CNS diseases $(n=9)$ : CNS vasculitis $(n=2)$, idiopathic recurrent myelitis $(n=2)$, and 1 case each of neuromyelitis optica, paraneoplastic cerebellitis, limbic encephalitis, Tolosa-Hunt syndrome, and chorioretinitis;

Group V - Immune-mediated neuropathies (IMN) $(n=10): 4$ cases of acute and 4 cases of chronic inflammatory demyelinating polyneuropathy, 1 case of acute motor axonal neuropathy and 1 case of polyneuropathy associated with trace amounts of IgM kappa paraprotein and anti-ganglioside IgM reactivity;

Group VI - Non-inflammatory nervous system diseases (NIND) $(n=79)$ : included a very wide and heterogeneous spectrum of diagnoses; more frequent were non-inflammatory (mostly diabetic) polyneuropathy $(n=9)$, discarthrosis, spinal canal stenosis and/or radiculopathy $(n=6)$, CNS tumors $(n=5)$, vertigo $(n=5)$, ischaemic stroke $(n=4)$, idiopathic facial nerve palsy $(n=3)$, motor neuron disease $(n=3)$, dementia $(n=3)$, and migraine $(\mathrm{n}=3)$;

Group VII - No evidence of organic neurological disorder (control group, $\mathrm{n}=15$ ): these patients presented mainly with mild mood disorders and/or psychosomatic problems.

For the analysis of the diagnostic value of CSFrestricted OCB tests, an MS group was created of patients fulfilling criteria for definite MS at the time of lumbar puncture $(n=28)$ plus patients presenting CIS who developed definite MS during the follow-up ( $n=9)$. The non-MS group consisted of patients in groups III, IV, V, VI and VII. Groups I, II, III, IV and V were considered inflammatory neurological diseases (IND), whereas groups VI and VII were considered "non-inflammatory".
Most patients were not treated by any immunomodulatory agents. However, we identified 30 patients who had received such treatment either at the time of the LP or less than six months prior to it: 7 patients in group I, 9 patients in group II, 2 patients in group III, 4 patients in group IV, 7 patients in group VI and 1 patient in group VII. One patient in the MS group was treated with natalizumab. Five patients were treated with intravenous methylprednisolone (IVMP) at the time of LP (first infusion at least one day before LP) and another five patients received IVMP 2-4 months prior to LP (two of them were still given low-dose oral steroids at the time of the LP). Another three patients were on low-dose oral steroids (in one case in combination with oral azathioprine). These treatments were given for MS or CIS-related symptoms. Another MS patient, suffering from type 1 diabetes with organ complications, received mycophenolate mofetil after previous renal/pancreas transplant, and one CIS patient received infliximab because of psoriasis. One patient with neuroborreliosis had been treated with intravenous dexamethasone several days prior to $L P$ because of radicular syndrome, and a patient with VZV-infection had received oral steroids for facial palsy. A patient with neuromyelitis optica had been treated with oral methylprednisolone and azathioprine and had received a series of plasma exchanges one month prior to LP. One patient with idiopathic recurrent myelitis received IVMP and both patients with CNS vasculitis received low-dose oral steroids. In the NIND group, two patients had been treated with interferon alfa-2a because of polycythaemia vera (one had received intravenous dexamethasone two weeks prior to the LP); two patients with suspected optic neuritis were treated with IVMP (final diagnoses were nasopharyngeal carcinoma with propagation into the orbit in one case and acute hypertonic neuroretinopathy in the other case), one patient received intravenous dexamethasone for radicular syndrome and two patients received low dose oral methylprednisolone in combination with sulfasalazine for rheumatoid arthritis and unspecified arthralgias, respectively. One patient in the control group too, had been treated with oral methylprednisolone because of suspected central limb monoparesis and minimal non-specific MRI changes (the final diagnosis was somatoform disorder).

In the exceptional cases of repeated lumbar punctures, the results of the first sample were used. Heavily bloodcontaminated CSFs were not used for the purpose of this study. Microscopic blood contamination was allowed, since no false positive results of the qualitative tests were expected.

$\mathrm{CSF}$ and sera were kept at $2-8{ }^{\circ} \mathrm{C}$ for up to one week for o-IgG test, and up to three weeks for o-fLC test. Repeated analyses of several samples showed consistent results during this time period. Samples were not frozen before OCB analysis.

o-IgG was detected by means of IEF followed by immunofixation using a commercial kit on Hydrasys instrument (Sebia, Évry Cedex, France, Cat. No. 4355). Standard amounts of IgG were applied. o-fLC were ana- 
lyzed in undiluted CSFs and paired sera diluted $1 / 100$ by IEF focusing followed by AIB as originally described by Sindic and Laterre ${ }^{3}$ and slightly modified by us ${ }^{19}$. This method combines the advantages of the previously described techniques of AIB ( ref. $^{20}$ ), glutaraldehyde fixation $^{21}$, biotin-( strept)avidin amplification ${ }^{22}$ and alkaline phosphatase detection ${ }^{23}$ to obtain maximum sensitivity. The only difference to our previous report consisted in the prolongation of the incubation time with biotinylated antibodies against free $\kappa$ and free $\lambda$ light chains to $105 \mathrm{~min}$, which improved the detection limit for free $\lambda$ up to 0.75 ng of monoclonal free $\lambda$ protein. o-IgGK and o-IgG $\lambda$ bands were analyzed as described ${ }^{19}$, but the amount of applied $\mathrm{IgG}$ was reduced to $12 \mathrm{ng}$. For logistic reasons and/or due to the shortage of antibodies, only 79 samples could be analyzed for the o-IgGK/IgG $\lambda$ pattern. The patient group described in this study included none of samples reported in our previous paper.

The results were classified as negative or positive based on the conventional criterion of $\geq 2$ CSF-restricted OCB for positivity. Classification into types 1-5 according to the international consensus for o-IgG (ref. ${ }^{24,25}$ ) was also performed but for the sake of simplicity is not reported in this paper. CSF-restricted bands were counted and if faint bands only were observed, this was noted. Analysis of receiver operating characteristic (ROC) curves was undertaken to find optimal cut-off values for the number of CSF-restricted OCB.

The predominance of free $\kappa$ or $\lambda$ bands was assessed on the basis of visual comparison of the blots, taking into account both the number and intensity of CSF restricted bands. However, at least twice as many bands of one type, compared to the other, were required for judging the predominance of one light chain type.
Statistical analysis was performed using MedCalc software version 11.4.4 (Frank Schoonjans, Belgium). Binomial exact confidence intervals were calculated for individual areas under the curve and the method of DeLong et al. ${ }^{26}$ was used to calculate the difference between two areas under the curve. Chi squared tests were used for categorical data.

\section{RESULTS}

Table 1 shows the proportions of samples positive for $o-I g G$, o-free $\kappa$ and o-free $\lambda$ in groups I-VII, using conventional criterion of at least 2 CSF-restricted OCB. The presence of o-IgG correlated with the presence of o-free $\kappa$ as well as o-free $\lambda$ (chi-square test, $P<0.0001$ ). Also, the presence of both o-fLC correlated with each other (chisquare test, $P<0.0001)$.

Subdividing CIS patients according to Miller et al. ${ }^{18}$ showed that the intrathecal humoral immune response strongly correlated with the MRI findings (chi-square test, $P=0.0081$ for o-IgG, $P<0.0001$ for o-free $\kappa$ and $P=0.0002$ for o-free $\lambda$ ). Only $2 / 6$ patients without at least one asymptomatic MRI lesion displayed CSF-restricted o-IgG (only two bands in both cases), and none displayed CSFrestricted o-fLC bands. In contrast, 32/36 patients with at least one asymptomatic MRI lesion displayed CSFrestricted o-IgG, 33 o-free $\kappa$, and 30 o-free $\lambda$ light chain bands. Nine CIS patients progressed to definite MS during the study. All of these patients had multiple MRI lesions, in 8 cases, CSF-restricted o-IgG as well as o-fLC (both o-free $\kappa$ and o-free $\lambda$ in 7 cases and o-free $\kappa$ only in the remaining case) and 1 patient was OCB-negative in all tests. In CNS infectious diseases, o-fLC were positive

Table 1. Proportion of patients positive for o- $\operatorname{lgG}$, o-free $\kappa$ and $\mathrm{o}$-free $\lambda$ in individual disease groups, using conventional criterion of $\geq 2$ CSF-restricted bands.

\begin{tabular}{lccccccc}
\hline Group & o-IgG & o-free $\kappa$ & o-free $\lambda$ & $\begin{array}{c}\text { At least 1 out } \\
\text { of the 3 tests }\end{array}$ & $\begin{array}{c}\text { At least 1 of } \\
\text { both fLC tests }\end{array}$ & $\begin{array}{c}\text { Both fLC } \\
\text { All tests posi- } \\
\text { tive }\end{array}$ \\
\hline I (MS; n=28) & 23 & 24 & 18 & 25 & 24 & 18 & 17 \\
& $(82.1 \%)$ & $(85.7 \%)$ & $(64.3 \%)$ & $(89.3 \%)$ & $(85.7 \%)$ & $(64.3 \%)$ & $(60.7 \%)$ \\
II (CIS; n=42) & 34 & 33 & 30 & 36 & 34 & 29 & 28 \\
& $(81.0 \%)$ & $(78.6 \%)$ & $(71.4 \%)$ & $(85.7 \%)$ & $(81.0 \%)$ & $(69.0 \%)$ & $(66.7 \%)$ \\
III (CNS infections; $\mathrm{n}=13)$ & 4 & 11 & 9 & 12 & 12 & 8 & 3 \\
& $(30.7 \%)$ & $(84.6 \%)$ & $(69.2 \%)$ & $(92.3 \%)$ & $(92.3 \%)$ & $(61.5 \%)$ & $(23.1 \%)$ \\
IV (OIND; n=9) & 5 & 7 & 6 & 7 & 7 & 8 & 5 \\
& $(55.6 \%)$ & $(77.8 \%)$ & $(66.7 \%)$ & $(77.8 \%)$ & $(77.8 \%)$ & $(88.9 \%)$ & $(55.6 \%)$ \\
V (IMN; n=10) & 1 & 5 & 3 & 6 & 5 & 3 & 0 \\
& $(10.0 \%)$ & $(50.0 \%)$ & $(30.0 \%)$ & $(60.0 \%)$ & $(50.0 \%)$ & $(30.0 \%)$ & $(0 \%)$ \\
VI (NIND; n=79) & 10 & 18 & 13 & 23 & 21 & 10 & 5 \\
VII (control group; $\mathrm{n}=15)$ & $(12.7 \%)$ & $(22.8 \%)$ & $(16.5 \%)$ & $(29.1 \%)$ & $(26.6 \%)$ & $(12.7 \%)$ & $(6.3 \%)$ \\
& $(6.7 \%)$ & $(20.0 \%)$ & $(0 \%)$ & $(20.0 \%)$ & $(20.0 \%)$ & $(0 \%)$ & 0 \\
\hline Total (n=196) & 78 & 101 & 79 & 112 & 106 & 76 & $(0 \%)$ \\
\hline
\end{tabular}

MS, multiple sclerosis; CIS, clinically isolated syndrome; OIND, other inflammatory CNS diseases; IMN, immune-mediated neuropathies; NIND, non-inflammatory nervous system diseases 
more frequently than o-IgG. The only o-fLC negative case of a patient with VZV ganglionitis was also negative for o-IgG, but pleocytosis and elevated anti-VZV antibody index were found.

For group IV, all OCB tests were negative in both patients with idiopatic myelitis. One case of CNS vasculitis was borderline positive for o-free $\kappa$, while other OCB tests were negative. In the other case of CNS vasculitis, all three OCB tests were positive, as in patients with paraneoplastic cerebellitis, neuromyelitis optica, Tolosa-Hunt syndrome, and chorioretinitis. In the case of limbic encephalitis, both o-fLC tests were positive but the o-IgG test was negative. 9 out of 10 cases of immune-mediated neuropathy (group V) were negative for CSF-restricted IgG bands, whereas 2 CSF-restricted IgG bands were found in the remaining case. Nevertheless, 5 cases were positive for CSF-restricted o-free $\kappa$ and 3 cases also for o-free $\lambda$ bands.

Within the NIND group, findings in 5 patients with CNS tumors are worth mentioning in detail. In 3/5 cases, CSF cytology was positive for atypical/malignant cells (highly atypical lymphocytes in 2 cases of the B-CLL and unspecified malignant cells in the case of malignant meningeal infiltration of unknown primary source), whereas only mild lymphocytic pleocytosis was present in the other 2 cases ( 1 case each of primary CNS lymphoma and glioblastoma). 3 cases were positive for o-IgG and all cases were positive for o-free $\kappa$ and 3 for o-free $\lambda$. An example of OCB findings in one patient of this group is shown in Fig. 1. Other positive cases in the NIND group com- prised a wide range of other diagnoses: ischaemic stroke and vestibular syndrome in two cases each and epilepsy, dementia, idiopathic facial palsy, ocular myositis, myasthenia gravis, vertigo, cervicocranial syndrome, cervical myelopathy, spinal stenosis, intervertebral disc herniation with radiculopathy, ischaemic mononeuropathy, toxic/diabetic polyneuropathy, fibromyalgias, headache in patient with monoclonal gammopathy of undetermined significance, tetany, mood disorder and somatoform disorder in one case each). Those cases without proven etiological diagnosis (i.e. with only symptom-related diagnoses) had features atypical for CIS, normal brain CT and/or MRI and no evidence of an infectious cause.

Analysis of samples positive for CSF-restricted o-fLC showed $\kappa$ fLC predominance in more than half of the cases (62 out of 106 o-fLC positive cases, i.e. 58.5\%), whereas free $\lambda$ predominance was rarely found ( 9 out of 106 cases, i.e. $8.5 \%)$. Free $\kappa / \lambda$ light chain predominance correlated well with the predominance of $\kappa / \lambda$ light chains in $\mathrm{IgG}$ (chi-square test, $P<0.0001$ ), although IgGk predominance was found somewhat less frequently than that of free $\kappa$ ( 13 out of 32 positive cases, i.e. $40.6 \%$ ).

Although there were similar frequencies of o-IgG and $o$-free $\kappa$ positivity in groups I and II, o-free $\kappa$ were positive more frequently than o-IgG not only in the other "inflammatory" groups III, IV and V, but also in the "non-inflammatory" groups VI and VII. This finding led us to search for an optimal criterion for positivity of individual tests.

Fig. 2 shows fewer CSF-restricted bands in patients in NIND and control groups than in IND patients. We there-

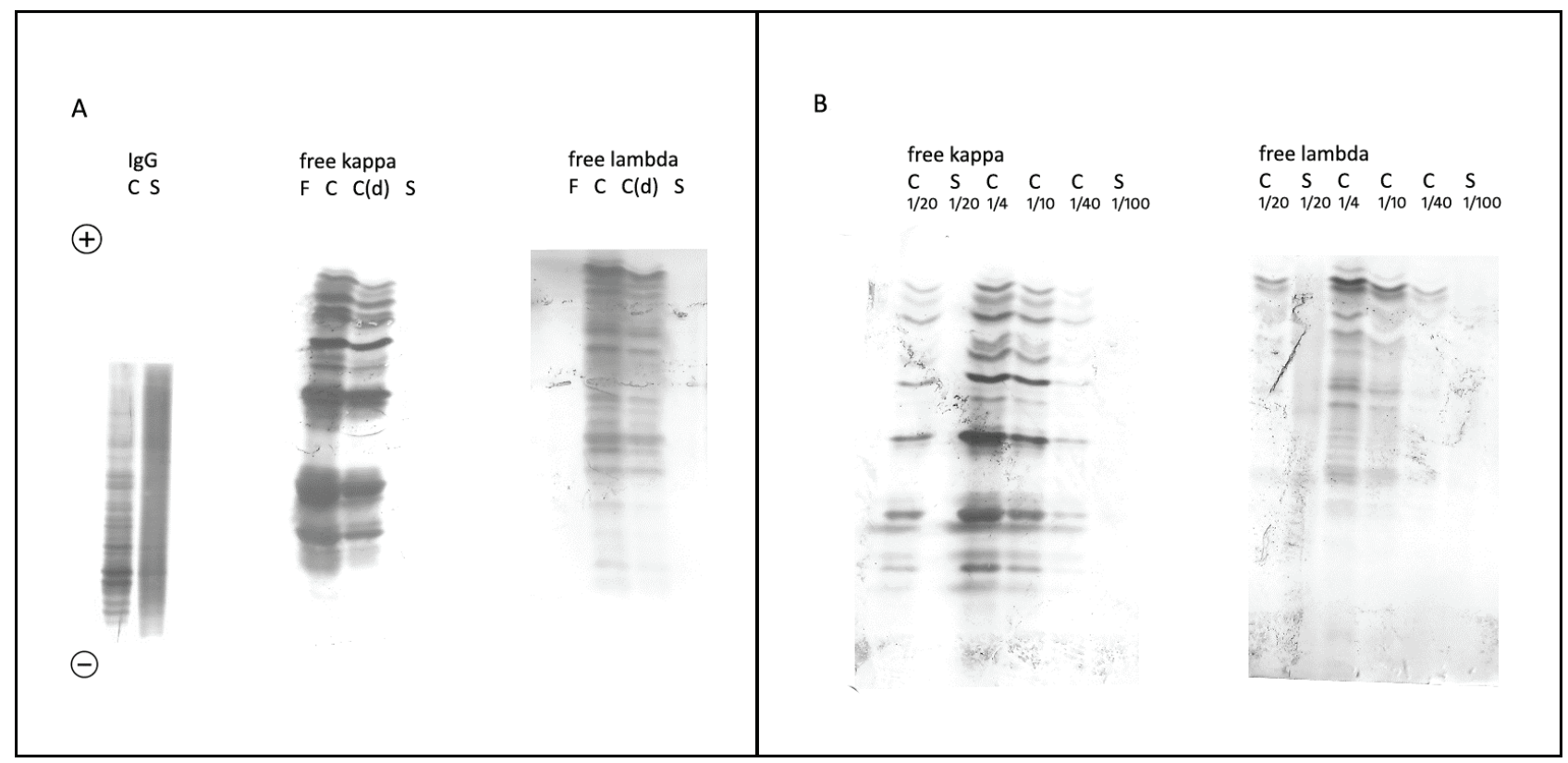

Fig. 1. Pronounced intrathecal IgG and fLC synthesis in the case of malignant meningeal infiltration.

C, cerebrospinal fluid; S, serum; F, Flebogamma (intravenous IgG preparation - negative control); (d), diluted 1/4. (A) CSFrestricted oligoclonal $\mathrm{IgG}$, free $\kappa$ and free $\lambda$ bands. For fLC analysis, CSF sample was examined neat as well as diluted $1 / 4$. (B) Dilution experiments showing CSF-restricted o-fLC bands even when CSF and serum were equally diluted (1/20). Higher concentration of CSF fLC than in serum can be assumed. Indeed, using Freelite kit on the SPA Analyzer (The Binding Site, Birmingham, United Kingdom), we measured CSF free $\kappa$ and free $\lambda 80.5 \mathrm{mg} / \mathrm{L}$ and $32.2 \mathrm{mg} / \mathrm{L}$, respectively, whereas serum free $\kappa$ and free $\lambda$ concentrations were $14.5 \mathrm{mg} / \mathrm{L}$ and $12.1 \mathrm{mg} / \mathrm{L}$, respectively. 


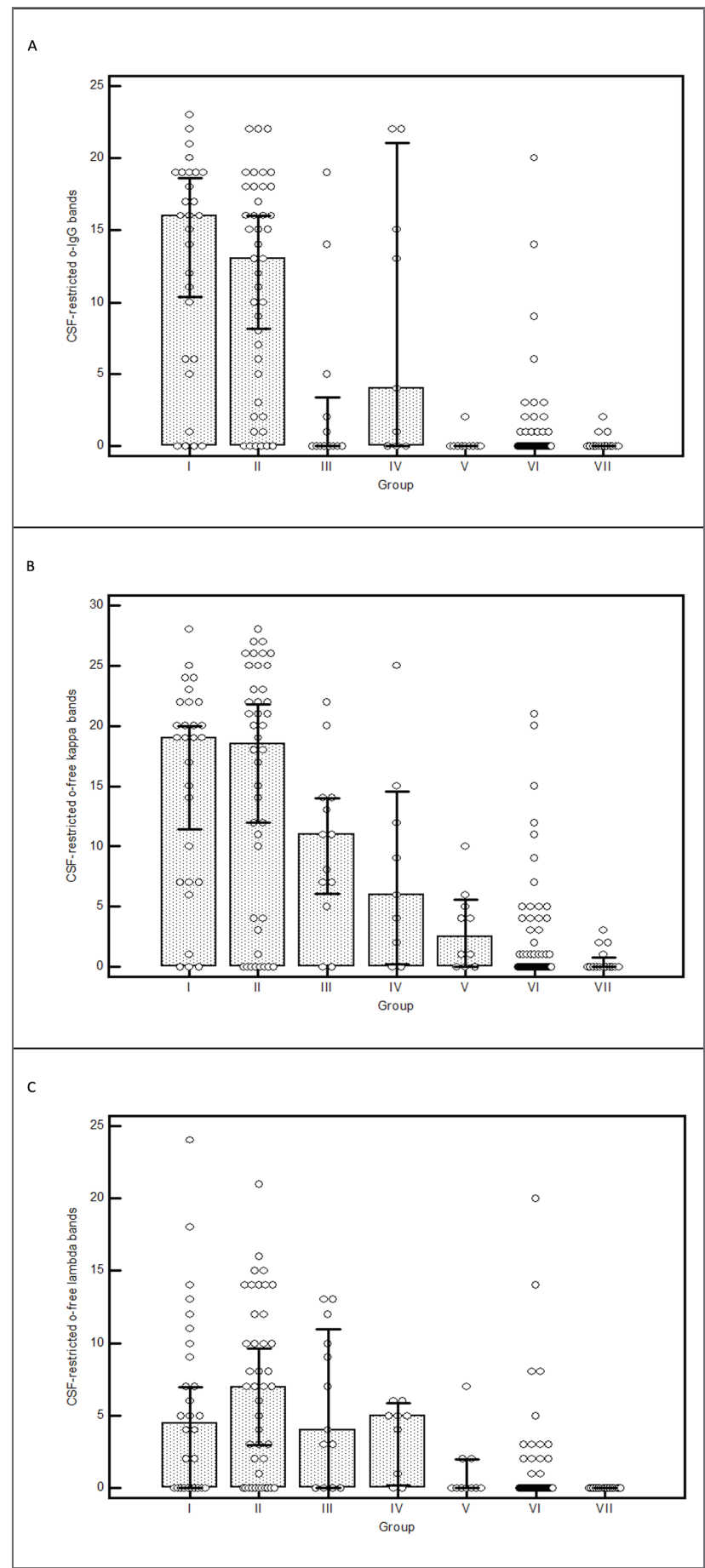

Fig. 2. Numbers of CSF-restricted oligoclonal bands in the individual disease groups. Bars for medians; error bars: $95 \%$ confidence intervals for medians

$\mathrm{I}$ = multiple sclerosis, $\mathrm{II}=$ clinically isolated syndrome, $\mathrm{III}=$ CNS infectious diseases, IV = other inflammatory CNS diseases, $\mathrm{V}=$ immune-mediated neuropathies, $\mathrm{VI}=$ non-inflammatory neurologic diseases, VII = control group. (A) oligoclonal IgG, (B) oligoclonal free $\kappa,(\mathrm{C})$ oligoclonal free $\lambda$.

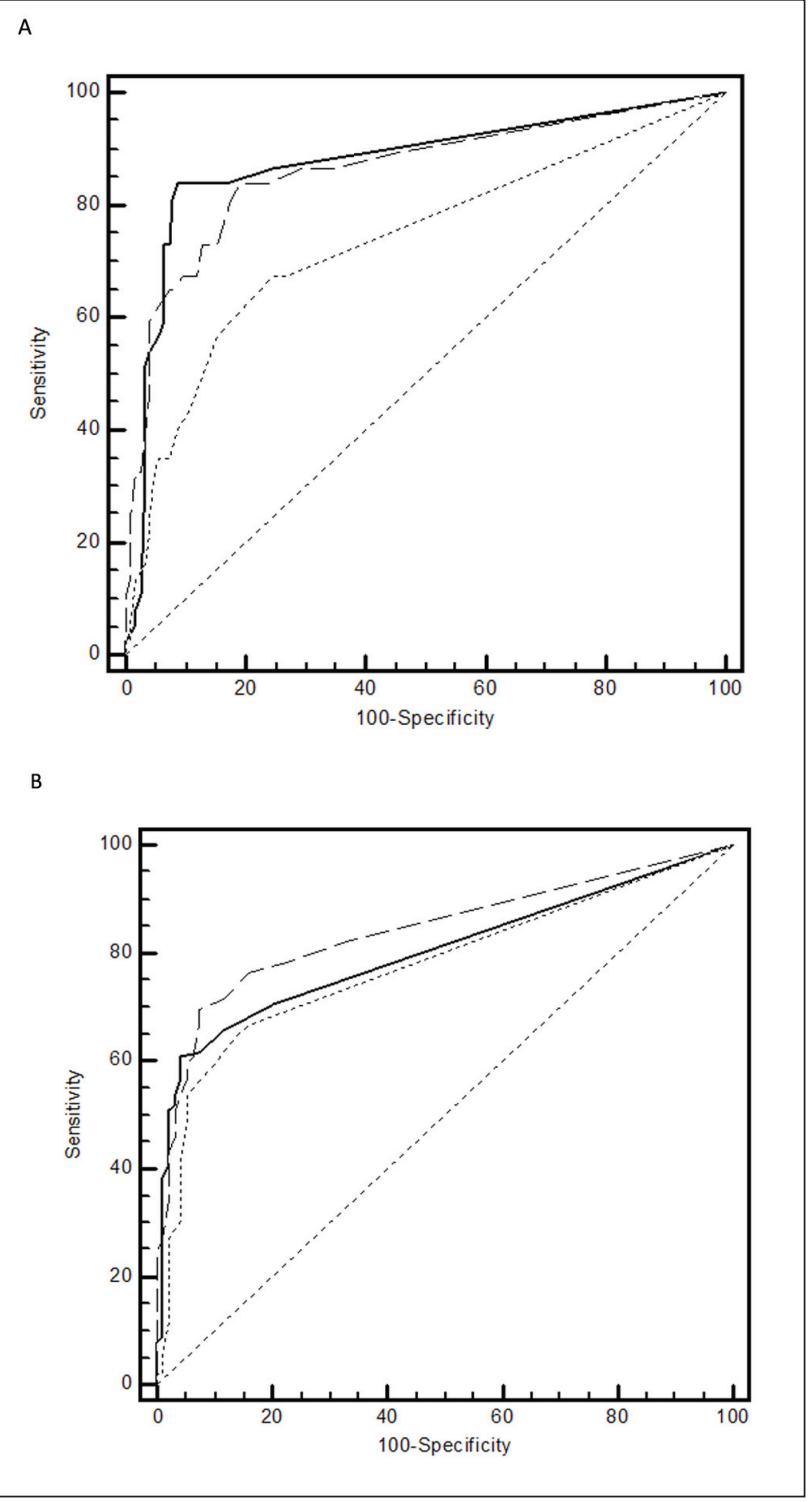

Fig. 3. Comparison of ROC curves for o-IgG (solid line), o-free $\kappa$ (dashed line) and o-free $\lambda$ (dotted line).

(A) For the diagnosis of multiple sclerosis. (B) For the diagnosis of inflammatory nervous system disease. For MS diagnosis, AUC values were 0.876 (95\% CI: 0.816-0.923), 0.862 (95\% CI: 0.800-0.911), and 0.739 (95\% CI: 0.664-0.804); for IND diagnosis, AUC values were 0.802 (95\% CI: 0.739-0.855), 0.843 (95\% CI: 0.784-0.891) and 0.777 (95\% CI: 0.712-0.833), for o-IgG, o-free $\kappa$ and o-free $\lambda$ respectively $(P<0.0001$ in all cases). Differences between areas were significant for o-IgG versus o-free $\lambda(P=0.0008)$ and o-free $\kappa$ versus o-free $\lambda(P=0.0026)$ in $\mathrm{A}$, and between o-free $\kappa$ versus o-free $\lambda(P=0.0059)$ in $\mathrm{B}$. 
Table 2. Sensitivity and specificity of CSF-restricted OCB for the diagnosis of MS or inflammatory neurologic disorder (IND) in general, using different cut-off values.

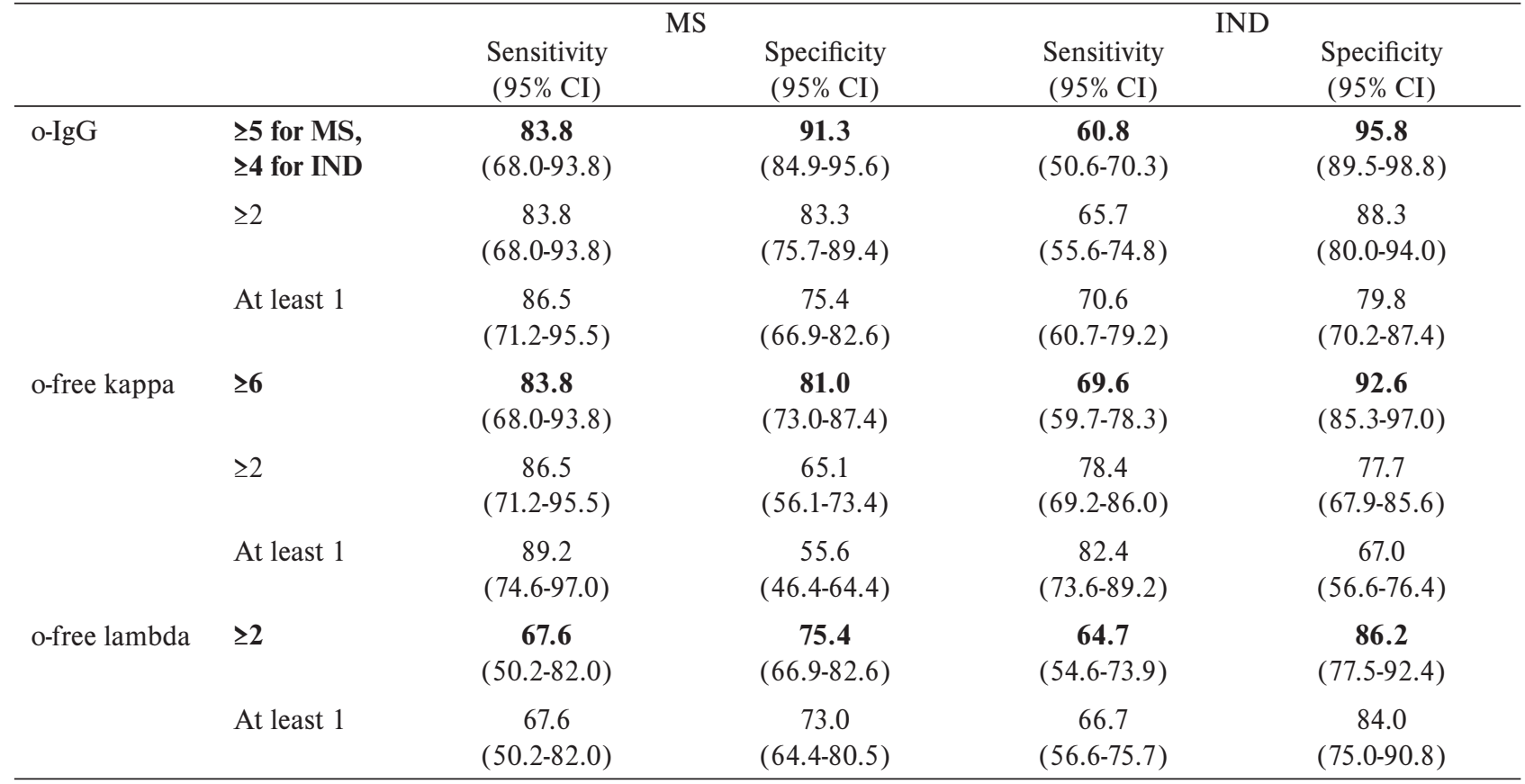

CI, confidence interval

Please note that MS group in Tables 3 and 4 is not identical with group I but comprises patients of group I ( $\mathrm{n}=28$ ) plus those patients presenting with CIS who developed definite MS during follow-up ( $n=9$ ). The IND group consists of patient groups I-V. Sensitivity is defined as the percentage of patients having MS or IND positive for CSF-restricted OCB; specificity for MS is defined as percentage of patients from groups III-VII without CSF-restricted OCB; specificity for IND is defined as percentage of patients from groups VI and VII without CSF-restricted OCB.

Calculated optimal cut-off values and appropriate sensitivities and specificities are indicated in bold.

Table 3. Sensitivity and specificity of CSF-restricted OCB combined tests for the diagnosis of MS or inflammatory neurologic disorder in general, using different cut-off values.

\begin{tabular}{llcccc}
\hline Combined test & Positivity criteria & \multicolumn{2}{c}{ MS } & \multicolumn{2}{c}{ IND } \\
& & Sensitivity & Specificity & Sensitivity & Specificity \\
\hline At least 1 OCB test + & Conventional ( $\geq 2$ CSF-restricted bands) & 89.2 & 59.5 & 84.3 & 72.3 \\
& Excluding weak bands & 86.5 & 65.1 & 77.5 & 74.5 \\
& Using optimal cut-off values & $\mathbf{8 9 . 2}$ & $\mathbf{6 9 . 8}$ & $\mathbf{7 7 . 5}$ & $\mathbf{8 0 . 9}$ \\
At least 1 fLC OCB test & Conventional ( $\geq 2$ CSF-restricted bands) & 86.5 & 61.9 & 80.4 & 74.5 \\
& Excluding weak bands & 83.8 & 66.7 & 74.5 & 75.5 \\
Both fLC OCB tests + & Using optimal cut-off values & $\mathbf{8 6 . 5}$ & $\mathbf{7 0 . 6}$ & $\mathbf{7 6 . 5}$ & $\mathbf{8 1 . 9}$ \\
& Conventional ( $\geq 2$ CSF-restricted bands) & 67.6 & 77.0 & 64.7 & 89.4 \\
All OCB tests + & Excluding weak bands & 62.2 & 84.9 & 53.9 & 94.7 \\
& Using optimal cut-off values & $\mathbf{6 7 . 6}$ & $\mathbf{8 5 . 7}$ & $\mathbf{5 7 . 8}$ & $\mathbf{9 6 . 8}$ \\
& Conventional ( $\geq 2$ CSF-restricted bands) & 64.9 & 89.7 & 52.0 & 94.7 \\
& Excluding weak bands & 59.5 & 92.1 & 43.1 & 96.8 \\
& Using optimal cut-off values & $\mathbf{6 4 . 9}$ & $\mathbf{9 2 . 9}$ & $\mathbf{4 8 . 0}$ & $\mathbf{9 7 . 9}$ \\
\hline
\end{tabular}

Optimal cut-off values for the number of CSF-restricted bands: $\geq 4$ for o-IgG; $\geq 6$ for free $\kappa$ and $\geq 2$ for free $\lambda$. For the sake of simplicity, minor difference in optimal cut-off values for o-IgG bands ( $\geq 5$ for MS and $\geq 4$ for IND) was not taken into account and the criterion of $\geq 4$ o-IgG bands was used for both groups. Sensitivities and specificities using optimal cut-off values are shown in bold. See also legend to Table 2 for details.

fore compared and analyzed ROC curves to determine the optimal cut-off values of the number of CSF-restricted bands, for the MS and IND diagnoses (Fig. 3).

An optimal cut-off for positivity was estimated to be $\geq 5 \mathrm{IgG}$ bands for MS and $\geq 4 \mathrm{IgG}$ bands for IND; for fLC bands, optimal cut-off values were the same for both purposes, namely $\geq 6$ for free $\kappa$ and $\geq 2$ for free $\lambda$.
The specificity and sensitivity of the tests using calculated optimal cut-off values or a conventional cut-off value of $\geq 2$ bands or at least 1 CSF-restricted band were compared (Tables 2 and 3 ).

We tested whether specificity could be increased if weak bands were ignored. This, however, decreased the sensitivity and failed to help increase the specificity (Table 3 ). In contrast, using optimal cut-off values helped 
increase specificity without compromising sensitivity. Noteworthy is that in $9 / 26$ cases with unexpected OCB positivity (patients in groups VI and VII), numbers of CSF-restricted OCB were below these cut-off values; in particular, all 3 patients from the control group positive for free $\kappa$ light chains had less than 5 free $\kappa$ bands and the single patient in this group positive for o-IgG only had 2 CSF-restricted o-IgG bands.

\section{DISCUSSION}

The main result of this study is the finding that optimizing the criteria for o-IgG and o-fLC positivity may increase specificity without compromising sensitivity. Although exact cut-off values require validation in an independent cohort and could depend on method used, the conclusion is that borderline findings of a few CSFrestricted bands should be interpreted with caution. For o-IgG, such findings have been found to be unreliable ${ }^{27}$ and mostly unrelated to the diagnosis of MS (ref. ${ }^{28}$ ). On the other hand, a few patients with such a finding, or even a single CSF-restricted band ${ }^{29,30}$, may have MS (ref. ${ }^{31}$ ). For this reason, we prefer to report borderline findings as such and not as negative. If there are numerous CSFrestricted bands, the value of band counting is questionable but bands should be counted in cases where only a few CSF-restricted bands are seen.

The frequencies of o-fLC and o-IgG positive results in MS are in agreement with other studies ${ }^{3,6,15,32}$. In CNS infections, CSF-restricted o-fLC bands were detected more frequently than CSF-restricted o-IgG bands. We speculate that intrathecal synthesis of o-fLC might precede detect- able intrathecal IgG synthesis; this should be confirmed in a larger study.

The immune-mediated neuropathies requires special attention as the absence of intrathecal humoral immune response is considered typical in these diseases. Indeed, only $1 / 10$ cases displayed a borderline positive o-IgG test. Nevertheless, half of these patients were positive for CSFrestricted o-fLC.

In the NIND group, the most striking finding was o-fLC positivity in CNS tumors. These findings undoubtedly do not represent 'false positivity' from the immunological point of view, but reflect the intrathecal humoral immune response that may be directed either against the tumor itself or, secondarily, against the damaged nervous tissue. The latter hypothesis was proposed by Arneth and Birklein to explain their findings of elevated free $\lambda$ concentrations in CSF in patients with cerebral ischaemia ${ }^{33}$, and although passionately discussed ${ }^{34,35}$, it requires further attention. Similar explanation is plausible for some of the other cases in the NIND group, although the possibility of unrecognized inflammatory cause cannot be completely ruled out.

All 6 CIS patients without at least one asymptomatic MRI lesion had negative OCB tests (except for 2 borderline o-IgG results) and none of them progressed to definite MS during the study. Nevertheless, no definite conclusions concerning the diagnostic use of CSF analysis in this subgroup of CIS patients can be made from our results due to small samples of the cases as well as absence of longer- follow-up data. Further studies are required to elucidate the value of o-IgG and o-fLC tests in this context. Although the diagnosis of MS, according to recent revision of $\mathrm{McD}$ onald criteria, does not require

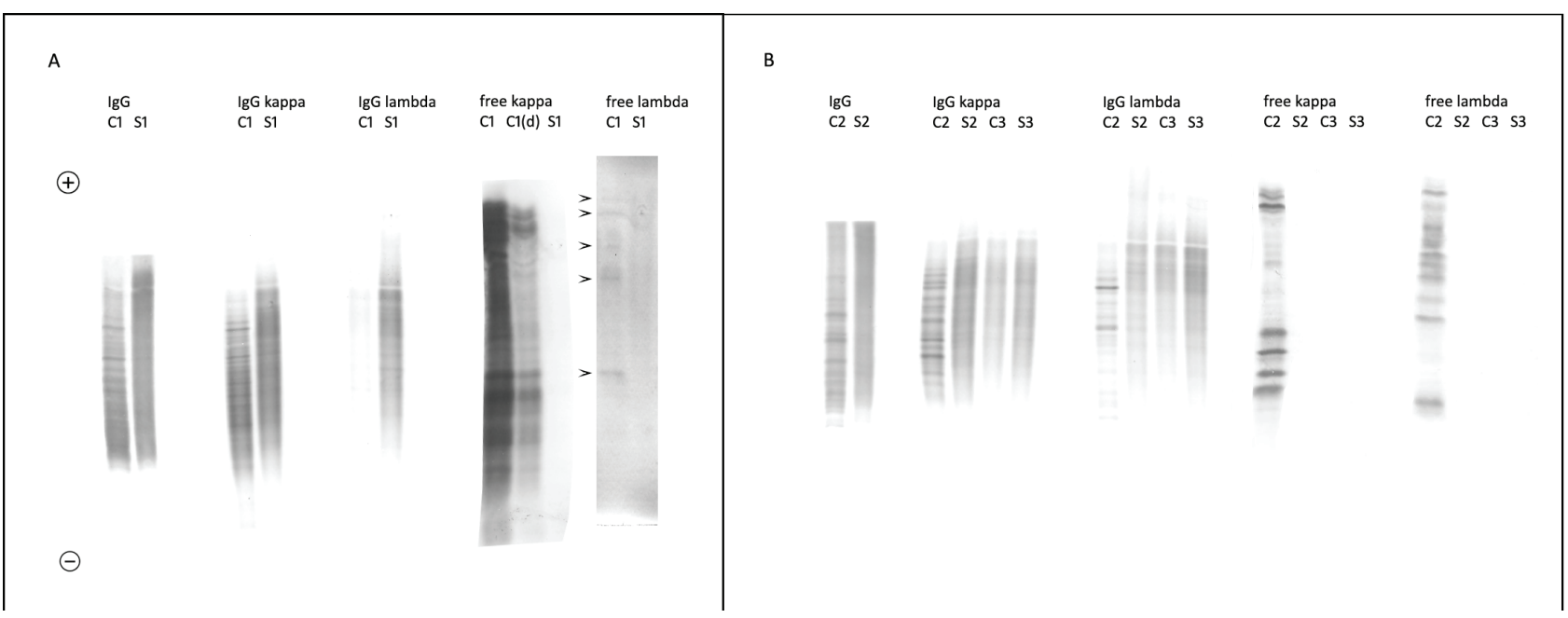

Fig. 4. Two patterns of CSF-restricted light chain distribution in MS patients.

C, cerebrospinal fluid; S, serum; (d), CSF diluted 1/5. Samples 1 and 2 (positive) originate from MS patients, whereas sample 3 (negative) is from the control group. (A) Clear predominance of $\kappa$ light chains can be seen in Sample 1 . Note that all IgG bands are of the $\kappa$ type, whereas almost no staining for $\operatorname{IgG} \lambda$ is visible in the CSF, suggesting highly elevated $\operatorname{IgG\kappa } / \operatorname{IgG} \lambda$ ratio. Also note that CSF had to be diluted $1 / 5$ for free $\kappa$ light chain analysis in order to make bands clearer, whereas only a few faint free $\lambda$ bands can be seen (arrowheads). (B) Both CSF-restricted IgG as well as free $\kappa$ and $\lambda$ light chains are present. Comparison of oligoclonal IgG pattern (IEF/IF) with IgGk/IgG $\lambda$ patterns (IEF/AIB) is difficult in this case, which can be expected considering the differences in the IEF procedures. 
CSF analysis (except for primary progressive MS fulfilling only 1 of 2 MRI criteria) (ref. ${ }^{13}$ ), most neurologists still recommend CSF examination as an important tool to exclude alternative diagnosis in CIS patients as well as to increase the predictive value for conversion to clinically definite MS (ref. ${ }^{36}$ ). It has been demonstrated that the presence of OCB considerably increases the risk of suffering a second attack even in MRI-negative patients ${ }^{37}$ and, in contrast, that as many as $50 \%$ of MRI-positive but OCB-negative CIS patients were finally diagnosed as having diseases other than MS during the follow-up ${ }^{38}$.

Different properties of fLC monomers and dimers have been reported, as well as a more specific CSF fLC profile for MS using sodium dodecyl sulfate-polyacrylamide gel electrophoresis (SDS-PAGE) separation ${ }^{40,41}$. Interestingly, we also found two types of CSF-restricted oligoclonal patterns in MS patients: one with a predominance of $\kappa$ light chains and the other with both CSF-restricted $\kappa$ as well as $\lambda$ light chains (Fig. 4). These two types may correspond to patients with the elevation of free $\kappa$ monomers and free $\lambda$ dimers in CSF in the study of Kaplan et al. ${ }^{41}$ On the other hand, free $\lambda$ predominance was rare in our study. It would be interesting to demonstrate whether $\kappa$ light chain predominance is associated with more or less aggressive course of the disease compared to balanced $\kappa$ and $\lambda$ light chain intrathecal production. The value of elevated CSF free $\kappa$ concentrations for predicting disability progression has already been reported ${ }^{41,42}$, but no ratio of CSF free $\kappa$ to CSF free $\lambda$ concentrations has been sought for. Further studies on similar relationships are warranted. Although the value of quantitative analysis prior to IEF is questioned by some authors ${ }^{43,44}$, it would be interesting to apply standardized amounts of fLC in both CSF and serum for IEF analysis. This would, however, require precise fLC quantification in both fluids that is difficult to achieve for the low concentrations that occur in normal CSFs (ref. ${ }^{45}$ ). Up to now, there is no commercially available method explicitly designated for fLC measurement in the CSF, and absolute values obtained by various ELISAs or nephelometric assays modified for fLC measurement in the CSF are quite discrepant ${ }^{5,33,46-50}$. Also, greater dilution of samples would require more sensitive, e.g. luminescent, detection of o-fLC on the membranes. We are not aware of any study using such an approach for o-fLC. Recently, quantitation of CSF free $\kappa$ light chains has been compared with o-IgG test in MS and CIS patients with encouraging results ${ }^{51-53}$. We believe that CSF fLC concentrations should be compared with the o-fLC test as well. It is known that qualitative analysis of o-IgG is more sensitive for the demonstration of intrathecal IgG synthesis than quantitative tests. However, this might not be true for fLC; indeed, correlation between quantitative and qualitative tests is much better than for $\operatorname{IgG}$, and no or only marginal increase in sensitivity has been achieved with the qualitative test compared to fLC quantitation in two studies ${ }^{3,5}$.

Unlike Kaplan et al. ${ }^{39,40}$, the specificity of our o-fLC test was lower than that of the o-IgG test. This might point to a more important role of fLC dimerization status (on which no information is available using IEF) than that of free $\kappa / \lambda$ proportion in the pathophysiology of MS, as it has been shown recently that fLC monomers and dimers are differentially secreted by plasma cells and functionally distinct ${ }^{54}$. Methods yielding combined information on both molecular weight and isoelectric point were described for fLC (ref. ${ }^{55}$ ), but these are technically more demanding and hardly applicable in routine clinical laboratories.

The antigen specificity of fLC (ref. ${ }^{3,54}$ ) should also be addressed in future studies. Only one study demonstrating antigen-specific fLC in the CSF was found ${ }^{56}$. These authors failed to demonstrate Toxoplasma gondii-specific fLC in AIDS patients with T. gondii encephalitis using IEF/AIB:although o-fLC were found in all patients; antigen-specific fLC were demonstrated by ELISA instead. The way Villar et al. described CSF-restricted lipid-specific oligoclonal IgM bands ${ }^{57}$, we can speculate whether CSF-restricted fLC could have the same specificity.

The main limitation of this study was patient selection. Although we attempted to investigate all consecutive samples sent for routine immunochemical CSF analyses, for logistic reasons this was not possible. Instead, after the publication of our previous study, we were frequently asked for o-fLC tests by clinicians. The neurologists were hence not blind to the results. However, it is rather unlikely that this could lead to misdiagnosis of MS since current MS diagnostic criteria ${ }^{13}$ do not usually rely on CSF findings. Inclusion of patients treated with immunomodulatory agents may also be considered problematic. Changes in o-IgG patterns after such therapies have been described by some authors ${ }^{58-60}$ but not others ${ }^{61,62}$. It should be noted, however, that the only natalizumab-treated patient in our study had numerous CSF-restricted o-IgG (o-IgGк only) as well as o-free $\kappa$ (but no o-free $\lambda$ ) bands, and that we found no significant difference either in the numbers of CSF-restricted o-IgG or o-fLC bands or in the proportions of positive samples in treated and untreated MS patients (data not shown). However, the study was not aimed at looking for these differences. Collection of larger data sets and analysis of paired pre- and post-treatment samples are clearly warranted to analyze the effect of treatment on o-IgG and o-fLC profile in detail.

There are two other concerns that might complicate the interpretation of the results. First, the inclusion of CIS patients who did not progress to definite MS in inflammatory neurological disease group remains speculative; it cannot be excluded that some of these patients suffered from a non-inflammatory condition.

Second, an optimal cut-off for the decision between negative or positive can vary for different OCB detection techniques.

One may argue that using sensitive IEF/AIB method for both o-IgG as well as for o-fLC detection would be more appropriate than comparison with routine o-IgG detection method. However, evaluation of an in-house method requires comparison with a widely used routine method rather than with another in-house method. In addition, we found no clinically significant difference between routine IEF with immunofixation (Sebia) and in-house IEF/AIB method for o-IgG detection ${ }^{27}$. 


\section{CONCLUSIONS}

For MS diagnosis, the CSF-restricted o-fLC test has similar sensitivity to the oligoclonal $\mathrm{IgG}$ test but lower specificity. For other inflammatory nervous system diseases, o-fLCs are more frequent than oligoclonal IgG. Detection of intrathecal fLC synthesis for routine clinical purposes is meaningful only when the o- $\operatorname{IgG}$ test is negative or borderline. Reporting borderline OCB results as positive, compromises the specificity of the test both for MS and IND and thus should be avoided. While we tried to assess the utility of the test for IND in general and to discuss unexpected findings in non-MS cases in more detail, further studies directed more specifically to the CIS and MS patients are warranted.

\section{ACKNOWLEDGEMENT}

The authors are indebted to Assoc. Prof. Kristian Safarcik, Head of the Dept. of Biomedical Sciences, University of Ostrava, and Dr. Zdenek Svagera, Head of the Dept. of Clinical Biochemistry, University Hospital Ostrava for their continuous support; to Ilona Faruzelova, Lenka Fürstova, Renata Maleckova, and Olga Michnova for performing routine o-IgG analyses; to Monika Hradecka for language corrections; to Frantisek Vsiansky for his advice on reporting the results of statistical analysis; and to Filip Chadzijski for his help in preparing figures for the manuscript.

Authorship contributions: DZ, PH, PK, OZ: study design; DZ, PK: evaluation of o-IgG and o-fLC tests; DZ, PH, RP, KR, IW, OZ: clinical data collection and management; DZ, PK, PH, RP, KR, IW, OZ: data analysis; DZ, PH, PK, OZ: manuscript drafting; DZ, PH, PK, RP, KR, IW, OZ: final approval.

Conflict of interest statement: The authors state that there are no conflicts of interest regarding the publication of this article.

\section{REFERENCES}

1. Sindic CJ, Van Antwerpen MP, Goffette S. The intrathecal humoral immune response: laboratory analysis and clinical relevance. Clin Chem Lab Med 2001;39:333-40.

2. Link H, Huang YM. Oligoclonal bands in multiple sclerosis cerebrospinal fluid: An update on methodology and clinical usefulness. J Neuroimmunol 2006;180:17-28.

3. Sindic CJ, Laterre EC. Oligoclonal free kappa and lambda bands in the cerebrospinal fluid of patients with multiple sclerosis and other neurological diseases. An immunoaffinity-mediated capillary blot study. J Neuroimmunol 1991;33:63-72.

4. Goffette S, Schluep M, Henry H, Duprez T, Sindic CJ. Detection of oligoclonal free kappa chains in the absence of oligoclonal lgG in the CSF of patients with suspected multiple sclerosis. J Neurol Neurosurg Psychiatry 2004;75:308-10.

5. Lamers KJ, de Jong JG, Jongen PJ, Kock-Jansen MJ, Teunesen MA, Prudon-Rosmulder EM. Cerebrospinal fluid free kappa light chains versus IgG findings in neurological disorders: qualitative and quantitative measurements. J Neuroimmunol 1995;62:19-25.

6. Krakauer M, Schaldemose Nielsen H, Jensen J, Sellebjerg F. Intrathecal synthesis of free immunoglobulin light chains in multiple sclerosis. Acta Neurol Scand 1998;98:161-5.
7. Bracco F, Gallo P, Menna R, Battistin L, Tavolato B. Free light chains in the CSF in multiple sclerosis. J Neurol 1987;234:303-7.

8. Gallo $\mathrm{P}$, Tavolato B, Bergenbrant $\mathrm{S}$, Sidén Á. Immunoglobulin light chain patterns in the cerebrospinal fluid. A study with special reference to the occurrence of free light chains in cerebrospinal fluid with and without oligoclonal immunoglobulin G. J Neurol Sci 1989;94:241-53.

9. Gallo P, Piccinno MG, De Rossi A, Pagni S, Amadori A, Chieco-Bianchi $\mathrm{L}$, Tavolato B. Free light chains of immunoglobulins in the cerebrospinal fluid of human immunodeficiency virus type 1-infected patients. J Neuroimmunol 1990;26:229-38.

10. Lolli F, Siracusa G, Amato MP, Fratiglioni L, Dal Pozzo G, Galli E, Amaducci L. Intrathecal synthesis of free immunoglobulin light chains and IgM in initial multiple sclerosis. Acta Neurol Scand 1991;83:239-43.

11. Vakaet A, Thompson EJ. Free light chains in the cerebrospinal fluid: an indicator of recent immunological stimulation. J Neurol Neurosurg Psychiatry 1985;48:995-8.

12. McLean BN, Miller D, Thompson EJ. Oligoclonal banding of IgG in CSF, blood-brain barrier function, and MRI findings in patients with sarcoidosis, systemic lupus erythematosus, and Behçet's disease involving the nervous system. J Neurol Neurosurg Psychiatry 1995;58:548-54.

13. Polman $\mathrm{CH}$, Reingold SC, Banwell B, Clanet $M$, Cohen JA, Filippi M, Fujihara K, Havrdova E, Hutchinson M, Kappos L, Lublin FD, Montalban X, O'Connor P, Sandberg-Wollheim M, Thompson AJ, Waubant E, Weinshenker B, Wolinsky JS. Diagnostic criteria for multiple sclerosis: 2010 revisions to the McDonald criteria. Ann Neurol 2011;69:292-302.

14. Mayringer I, Timeltaler B, Deisenhammer F. Correlation between the IgG index, oligoclonal bands in CSF, and the diagnosis of demyelinating diseases. Eur J Neurol 2005;12:527-30.

15. Bourahoui A, de Seze J, Guttierez R, Onraed B, Hennache B, Ferriby $D$, Stojkovic T, Vermersch P. CSF isoelectrofocusing in a large cohort of MS and other neurological diseases. Eur J Neurol 2004;11:525-9.

16. Wurster $U$. The clinical significance of an intrathecal monoclonal immunoglobulin band: A follow up study. Letter to the Editor. Neurology 2004;62:1237.

17. Lourenco P, Shirani A, Saeedi J, Oger J, Schreiber WE, Tremlett H. Oligoclonal bands and cerebrospinal fluid markers in multiple sclerosis: associations with disease course and progression. Mult Scler 2012;19:577-84.

18. Miller DH, Weinshenker BG, Filippi M, Banvell BL, Cohen JA, Freedman MS, Galetta SL, Hutchinson M, Johnson RT, Kappos L, Kira J, Lublin FD, McFarland HF, Montalban X, Panitch H, Richert JR, Reingold SC, Polman CH. Differential diagnosis of suspected multiple sclerosis: a consensus approach. Mult Scler 2008;14:1157-74.

19. Zeman D, Hradílek P, Švagera Z, Mojžíšková E, Woznicová I, Zapletalová O.. Detection of oligoclonal IgG kappa and IgG lambda using Hevylite antibodies. Comparison with oligoclonal free light chain pattern. Fluids Barriers CNS 2012;9:5.

20. Knisley KA, Rodkey LS. Affinity immunoblotting. High resolution isoelectric focusing analysis of antibody clonotype distribution. J Immunol Methods 1986;95:79-87.

21. Ikegati N, Kennet RH. Glutaraldehyde fixation of the primary antibody-antigen complex on nitrocelulose paper increases the overall sensitivity of immunoblot assay. J Immunol Methods 1989;124:20510.

22. Olsson T, Kostulas V, Link H. Improved detection of oligoclonal lgG in cerebrospinal fluid by isoelectric focusing in agarose, doubleantibody peroxidase labeling, and avidin-biotin amplification. Clin Chem 1984;30:1246-9.

23. Sádaba MC, González-Porqué P, Masjuan J, Álvarez-Cermeño JC, Bootello A, Villar LM. An ultrasensitive method for the detection of oligoclonal IgG bands. J Immunol Methods 2004;284:141-5.

24. Andersson M, Alvarez-Cermeño J, Bernardi G, Cogato I, Fredman $P$, Frederiksen J, Fredrikson S, Gallo P, Grimaldi LM, Grønning M, Keir G, Lamers K, Link H, Magalhães A, Massaro AR, Öhman S, Reiber H, Rönnbäck L, Schluep M, Schuller E, Sindic CJ, Thompson EJ, Trojano M, Wurster U. Cerebrospinal fluid in the diagnosis of multiple sclerosis: a consensus report. J Neurol Neurosurg Psychiatry 1994;57:897902.

25. Freedman MS, Thompson EJ, Deisenhammer F, Giovannoni G, Grimsley G, Keir G, Öhman S, Racke MK, Sharief M, Sindic CJ, 
Sellebjerg F, Tourtellotte WW. Recommended standard of cerebrospinal fluid analysis in the diagnosis of multiple sclerosis. Arch Neurol 2005;62:865-70.

26. DeLong ER, DeLong DM, Clarke-Pearson DL. Comparing the areas under two or more correlated receiver operating characteristic curves: a non-parametric approach. Biometrics 1988;44:837-45.

27. Novackova L, Zeman D. Detection of oligoclonal IgG bands in cerebrospinal fluid and serum: comparison between commercial immunofixation method and home-made affinity immunoblotting method and evaluation of interobserver agreement. Klin Biochem Metab 2011;19:229-33.

28. Lin MW, Suan D, Lenton K, Henniker T, Burke T, Vucic S, Fulcher DA Differentiating patterns of oligoclonal banding in the cerebrospinal fluid improves diagnostic utility for multiple sclerosis. Pathology 2012;44:248-50.

29. Davies G, Keir G, Thompson EJ, Giovannoni G. The clinical significance of an intrathecal monoclonal immunoglobulin band: a followup study. Neurology 2003;60:1163-6.

30. Franciotta D, Bergamaschi R, Amato MP, Zardini E, Persico A, Portaccio E, Lolli F. Clinical correlations of CSF single IgG bands. J Neurol 2005;252:1274-5.

31. Absalom S, Shah S. Differentiating patterns of oligoclonal banding in the cerebrospinal fluid and the diagnosis of multiple sclerosis. Pathology 2013;45:441-2.

32. Dobson R, Ramagopalan S, Davis A, Giovannoni G. Cerebrospinal fluid oligoclonal bands in multiple sclerosis and clinically isolated syndromes: a meta-analysis of prevalence, prognosis and effect of latitude. J Neurol Neurosurg Psychiatry 2013;84:909-14.

33. Arneth B, Birklein F. High sensitivity of free lambda and free kappa light chains for detection of intrathecal immunoglobulin synthesis in cerebrospinal fluid. Acta Neurol Scand 2009;119:39-44.

34. Reiber $\mathrm{H}$. Free light chains in CSF - pushing a method with biased in terpretations. Letter to the editor. Acta Neurol Scand 2009;120:445-6.

35. Arneth B. Author's response to professor Reiber's second letter concerning our article: High sensitivity of free lambda and free kappa light chains for the detection of intrathecal immunoglobulin synthesis in cerebrospinal fluid. Acta Neurol Scand 2009; 120:451-2.

36. Tumani $H$, Deisenhammer F, Giovannoni G, Gold R, Hartung HP, Hemmer B, Hohlfeld R, Otto M, Stangel M, Wildemann B, Zettl UK. Revised McDonald kriteria: the persisting importance of cerebrospinal fluid analysis. Letter to the Editor. Ann Neurol 2011;70:520.

37. Tintoré $M$, Rovira A, Río J, Tur C, Pelayo R, Nos C, Téllez N, Perkal $\mathrm{H}$, Comabella M, Sastre-Garriga J, Montalban X. Do oligoclonal bands add information to MRI in first attacks of multiple sclerosis? Neurology 2008;70:1079-83.

38. Zipoli V, Hakiki B, Portaccio E, Lolli F, Siracusa G, Giannini M, Panton L, Pescini F, Sorbi S, Amato MP. The contribution of cerebrospinal fluid oligoclonal bands to the early diagnosis of multiple sclerosis. Mult Scler 2009;15:472-8.

39. Kaplan B, Aizenbud BM, Golderman S,Yaskariev R, Sela BA. Free light chain monomers in the diagnosis of multiple sclerosis. J Neuroimmunol 2010;229:263-71.

40. Kaplan B, Golderman S, Yahalom G, Yeskaraev R, Ziv T, Aizenbud BM, Sela BA, Livneh A. Free light chain monomer-dimer patterns in the diagnosis of multiple sclerosis. J Immunol Methods 2013;390:74-80.

41. Rudick RA, Medendorp SV, Namey M, Boyle S, Fischer J. Multiple sclerosis progression in a natural history study: Predictive value of cerebrospinal fluid free kappa light chains. Mult Scler 1995;1:150-5.

42. Rinker JR, Trinkaus K, Cross AH. Elevated CSF free kappa light chains correlate with disability prognosis in multiple sclerosis. Neurology 2006;67:1288-90.

43. Thompson EJ. Quality versus quantity: Which is better for cerebrospinal fluid lgG? Clin Chem 2004;50:1721-2.

44. Keir G, Barrio S, Thompson EJ. Discrepancies between qualitative and quantitative analysis of cerebrospinal fluid lgG: densitometric comparison of nephelometry-silver versus immuno-enzymes. Ann Clin Biochem 2008;45:404-8.

45. Nakano T, Miyazaki S, Takahashi H, Matsumori A, Maruyama T, Komoda T, Nagata A. Immunochemical quantification of free immunoglobulin light chains from an analytical perspective. Clin Chem Lab Med 2006:44:522-32.
46. Fagnart OC, Sindic CJM, Laterre C. Free kappa and lambda light chain levels in the cerebrospinal fluid of patients with multiple sclerosis and other neurological diseases. J Neuroimmunol 1988;19:119-32.

47. Lolli F, Amaducci L. Measurement of free kappa immunoglobulin light chains in the cerebrospinal fluid by a competitive avidin-biotin ELISA. Clin Chim Acta 1989;182:229-34.

48. Fischer C, Arneth B, Koehler J, Lotz J, Lackner KJ. Kappa free light chains in cerebrospinal fluid as markers of intrathecal immunoglobulin synthesis. Clin Chem 2004;50:1809-13.

49. Desplat-Jégo S, Feuillet L, Pelletier J, Bernard D, Chériff AA, Boucraut J. Quantification of immunoglobulin free light chains in cerebrospinal fluid by nephelometry. J Clin Immunol 2005;25:338-45.

50. Presslauer S, Milosavljevic D, Brücke T, Bayer P, Hübl W. Elevated levels of kappa free light chains in CSF support the diagnosis of multiple sclerosis. J Neurol 2008;255:1508-14.

51. Villar LM, Espiño M, Costa-Frossard L, Muriel A, Jiménez J, ÁlvarezCermeño JC. High levels of cerebrospinal fluid free kappa chains predict conversion to multiple sclerosis. Clin Chim Acta 2012;413:1813-6.

52. Presslauer S, Milosavljevic D, Huebl W, Parigger S, Schneider-Koch G, Bruecke T. Kappa free light chains: diagnostic and prognostic relevance in MS and CIS. PLoS One 2014;9(2):e89945.

53. Senel M, Tumani H, Lauda C, Presslauer S, Mojib-Yezdani R, Otto M, Brettschneider J. Cerebrospinal fluid immunoglobulin kappa light chain in clinically isolated syndrome and multiple sclerosis. PLoS One 2014;9(4):e88680.

54. Groot Kormelink T, Blokhuis BR, Kil L, Hendriks R, Redegeld FA. Immunoglobulin free light chain monomers and dimers are differentially secreted by plasma cells and are functionally distinct. In: Groot Kormelink T. Immunoglobulin free light chains in inflammatory diseases. New findings on FLCs fitted into current concepts of immune regulation. Thesis. GVO/Ponson \& Looijen B.V., Ede, The Netherlands 2012:187-203.

55. Kaplan B, Ramirez-Alvarado M, Dispenzieri A, Zeldenrust SR, Leung N, Livneh A, Gallo G. Isolation and biochemical characterization of plasma monoclonal free light chains in amyloidosis and multiple myeloma: a pilot study of intact and truncated forms of light chains and their charge properties. Clin Chem Lab Med 2008;46:335-41.

56. Contini C, Gainardi E, Cultrera R, Seraceni S, Castellazi M, Peyron F, Granieri E. Evidence of cerebrospinal fluid free kappa light chains in AIDS patients with Toxoplasma gondii encephalitis. J Neuroimmunol 2000;108:221-6.

57. Villar LM, Sádaba MC, Roldán E, Masjuan J, González-Porqué $P$, Villarrubia N, Espiño M, García-Trujillo JA, Bootello A, AlvarezCermeño JC. Intrathecal synthesis of oligoclonal IgM against myelin lipids predicts an aggressive disease course in MS. J Clin Invest 2005; 115:187-94.

58. Anderson TJ, Donaldson IM, Sheat JM, George PM. Methylprednisolone in multiple sclerosis exacerbation: changes in CSF parameters. Aust N Z J Med 1990;20:794-7.

59. Mares J, Muchova B, Herzig R, Urbanek K. Changes in the pattern of oligoclonal bands in the cerebrospinal fluid of patients with multiple sclerosis during therapy with beta interferons, and glatiramer acetate respectively. Pilot study. Ces a Slov Neurol Neurochir 2003;66/99:118-20

60. Von Glehn F, Farias AS, de Oliveira AC, Damasceno A, Longhini AL, Oliveira EC, Damasceno BP, Santos LM, Brandao CO. Disappearance of cerebrospinal fluid oligoclonal bands after natalizumab treatment of multiple sclerosis patients. Mult Scler 2012;18:1038-41.

61. Tourtellotte WW. The cerebrospinal fluid in multiple sclerosis. In: Vinken JP, Bruyn GW, Klawans HL, Koetsier JC, editors. Handbook of Clinical Neurology Vol. 47. Demyelinating diseases. Rev. ser. 3. Amsterdam: Elsevier; 1985. p. 79-130.

62. Rudick RA, Cookfair DL, Simonian NA, Ransohoff RM, Richert JR, Jacobs LD, Herndon RM, Salazar AM, Fischer JS, Granger CV, Goodkin DE, Simon JH, Bartoszak DM, Bourdette DN, Braiman J, Brownscheidle CM, Coats ME, Cohan SL, Dougherty DS, Kinkel RP, Mass MK, Munchsauer FE, O'Reilly K, Priore RL, Pullicino PM, Scherokman BJ, Wende K, Weinstock-Guttman B, Whitham RH, The Multiple Sclerosis Collaborative Research Group. Cerebrospinal fluid abnormalities in a phase III trial of Avonex (IFN-beta 1a) for relapsing multiple sclerosis. J Neuroimmunol 1999;93:8-14. 